\title{
Singularity Analysis of a Six-dof Parallel Manipulator using Grassmann-Cayley Algebra and Gröbner Bases
}

\author{
Stéphane Caro ${ }^{1}$, Guillaume Moroz ${ }^{1}$, Thibault Gayral ${ }^{1,2}$, Damien Chablat ${ }^{1}$ and \\ Chao Chen ${ }^{2}$
}

\begin{abstract}
The subject of this paper deals with the singularity analysis of a sixdof three-legged parallel manipulator for force-feedback interface. To this end, a geometric condition for the manipulator singularities is obtained by means of Grassmann-Cayley algebra; the parallel singularities of the manipulator are computed using Jacobian and Gröbner basis. As a result, the algebraic relations of the singularities satisfied by the orientation variables are reported. Finally, the parallel singularities of the manipulator are plotted in its orientation workspace.
\end{abstract}

\section{Introduction}

The challenge in Virtual Reality (VR) today is to develop "transparent" haptic interface. VR aims at creating high-fidelity reproductions of a real environment, where a user perceives the illusion of being in this environment and interacts with it to perform specific tasks in an effective and comfortable way [1]. Haptics is critical in VR applications, where the user actively manipulates the simulated world, such as in surgical simulators [2]. There are two main classes of haptic devices: admittance devices and impedance devices [3]. Admittance devices sense the force applied by the operator and then control the operator's position. They are often more expensive because of the cost of the required force sensors, and more complex because of the weight of the force sensors. Impedance devices, also termed force feedback devices, are simpler, cheaper and more common. The fidelity in VR requires light and stiff interfaces. In this vein, Chen et al. developed a new six-dof three-legged parallel manipulator for force-feedback interface [4]. The advantage of the proposed device is that all motors are mounted on the ground. This feature significantly reduces the

\footnotetext{
${ }^{1}$ Institut de Recherche en Communications et Cybernétique de Nantes, France, e-mail: \{stephane.caro, guillaume.moroz, damien.chablat\}@irccyn.ec-nantes.fr

${ }^{2}$ Department of Mechanical and Aerospace Engineering, Monash University, Australia, e-mail: thibault.gayral@ens-cachan.org, chao.chen@monash.edu
} 
overall mass and inertia of the moving parts, which will eventually yield a more transparent haptic device.

Here, we focus on the singularity analysis of the foregoing manipulator by using Grassmann-Cayley algebra (GCA) and Gröbner bases (GB). Singularities can be found using either numeric, symbolic or geometric methods. For Gough-Stewart parallel manipulators, the rows of the backward Jacobian matrix are the Plücker coordinate vectors of six finite lines that are the lines of action of six actuation forces applied by the actuators to the moving platform. The parallel singularities of such manipulators that Merlet [5] analyzed using Grassmann line geometry occur when those lines become linearly dependent. Ben-Horin and Shoham analyzed the parallel singularities of six-dof parallel manipulators using GCA [6, 7]. Kanaan et al. [8] enlarged the application of GCA to lower-mobility manipulators, in which the rows of the backward Jacobian are either finite lines (zero pitch wrenches) or infinite lines (infinite pitch wrenches). These wrenches, also known as governing lines, are actuation and constraint wrenches applied to the moving platform.

This paper is organized as follows. Section 2 describes the manipulator under study. Sections 3 and 4 are devoted to its singularity analysis with GrassmannCayley algebra and Gröbner bases, respectively. Finally, the parallel singularities of the manipulator are plotted in its orientation workspace.

\section{Manipulator Description}

Figure 1 illustrates the parallel manipulator under study, which is a simplified kinematic version of the manipulator proposed in [4]. It is composed of an equilateral moving platform connected to the base by means of three identical legs. Each leg is composed of three orthogonal prismatic joints and one spherical joint, the first two prismatic joints being actuated. $\mathrm{P}$ stands for a prismatic joint whereas $\mathrm{S}$ stands for a spherical joint. An underline letter denotes an actuated joint. As a consequence, the manipulator is named 3-PPPS manipulator and provides six-degree-of-freedom motions, i.e., three translations and three rotations.

\subsection{Parameterization}

Let $C_{1}, C_{2}$ and $C_{3}$ be the corners of the moving platform (MP) of side length $r$. Let $\mathscr{F}_{p}\left(C_{p}, X_{p}, Y_{p}, Z_{p}\right)$ be the frame attached to the moving platform, its origin $C_{p}$ being the centroid of the MP. $Y_{p}$ is parallel to line $\left(C_{2} C_{3}\right)$ and $Z_{p}$ is normal to the MP. Accordingly,

$$
\mathbf{c}_{1 p}=\left[\begin{array}{c}
2 r \sqrt{3} / 6 \\
0 \\
0
\end{array}\right], \mathbf{c}_{2 p}=\left[\begin{array}{c}
-r \sqrt{3} / 6 \\
r / 2 \\
0
\end{array}\right], \mathbf{c}_{3 p}=\left[\begin{array}{c}
-r \sqrt{3} / 6 \\
-r / 2 \\
0
\end{array}\right]
$$






Fig. 1 The 3-PPPS manipulator

are the Cartesian coordinate vectors of points $C_{1}, C_{2}$ and $C_{3}$ expressed in $\mathscr{F}_{p}$. Likewise, let $\mathscr{F}_{b}(O, X, Y, Z)$ be the frame attached to the base and

$$
\mathbf{a}_{1 b}=\left[\begin{array}{c}
x_{1} \\
y_{1} \\
0
\end{array}\right], \mathbf{a}_{2 b}=\left[\begin{array}{c}
0 \\
y_{2} \\
z_{2}
\end{array}\right], \mathbf{a}_{3 b}=\left[\begin{array}{c}
x_{3} \\
0 \\
z_{3}
\end{array}\right]
$$

be the Cartesian coordinate vectors of points $A_{1}, A_{2}$ and $A_{3}$.

\subsection{Orientation Space}

The orientation space can be fully represented with the variables $\left(Q_{2}, Q_{3}, Q_{4}\right)$, a subset of the quaternions coordinates. Indeed, the quaternions represent the rotations of the platform with a rotation axis $\mathbf{u}$ and an angle $\theta$. The relation between the quaternions and the axis and angle representation can be found in [9]: 
$Q_{1}=\cos (\theta / 2), Q_{2}=u_{x} \sin (\theta / 2), Q_{3}=u_{y} \sin (\theta / 2), Q_{4}=u_{z} \sin (\theta / 2)$

where $u_{x}^{2}+u_{y}^{2}+u_{z}^{2}=1$ and $0 \leq \theta \leq \pi$.

Thus, each rotation can be mapped onto a point of the unit ball in the space defined by the variables $\left(Q_{2}, Q_{3}, Q_{4}\right)$ with the following bijection:

$$
\begin{aligned}
\Phi: \mathscr{S} \times] 0, \pi] \rightarrow \mathscr{B} \backslash\left\{\left(\begin{array}{l}
0 \\
0 \\
0
\end{array}\right)\right\} \\
\left(\begin{array}{l}
u_{x} \\
u_{y} \\
u_{z}
\end{array}\right), \theta \mapsto\left(\begin{array}{l}
Q_{2}:=u_{x} \sin (\theta / 2) \\
Q_{3}:=u_{y} \sin (\theta / 2) \\
Q_{4}:=u_{z} \sin (\theta / 2)
\end{array}\right)
\end{aligned}
$$

where $\mathscr{S}$ is the unit sphere in a 3 -dimension space, and $\mathscr{B}$ is the closed unit ball.

When $\theta$ is equal to zero, the corresponding rotation matrix is the identity that does not depend on the rotation axis $\mathbf{u}$. It also maps to the center of $\mathscr{B}$ in the quaternions representation.

\subsection{Geometric Model}

Let $\mathbf{c}_{b}=\left[\begin{array}{lll}c_{x} & c_{y} & c_{z}\end{array}\right]^{T}$ be the Cartesian coordinate vector of point $C$, the centroid of the MP, expressed in $\mathscr{F}_{b}$. The following equations characterize the geometric model of the 3-PPPS manipulator:

$$
\begin{aligned}
c_{x}-1 / 3 \sqrt{3} Q_{1}{ }^{2}-1 / 3 \sqrt{3} Q_{2}{ }^{2}+1 / 6 \sqrt{3}-Q_{2} Q_{3}+Q_{1} Q_{4}-x_{1} & =0 \\
c_{y}-1 / 3 \sqrt{3} Q_{2} Q_{3}-1 / 3 \sqrt{3} Q_{1} Q_{4}-Q_{1}{ }^{2}-Q_{3}{ }^{2}+1 / 2-y_{1} & =0 \\
c_{y}-1 / 3 \sqrt{3} Q_{2} Q_{3}-1 / 3 \sqrt{3} Q_{1} Q_{4}+Q_{1}{ }^{2}+Q_{3}{ }^{2}-1 / 2-y_{2} & =0 \\
c_{z}-1 / 3 \sqrt{3} Q_{2} Q_{4}+1 / 3 \sqrt{3} Q_{1} Q_{3}+Q_{3} Q_{4}+Q_{1} Q_{2}-z_{2} & =0 \\
c_{x}+2 / 3 \sqrt{3} Q_{1}{ }^{2}+2 / 3 \sqrt{3} Q_{2}{ }^{2}-1 / 3 \sqrt{3}-x_{3} & =0 \\
c_{z}+2 / 3 \sqrt{3} Q_{2} Q_{4}-2 / 3 \sqrt{3} Q_{1} Q_{3}-z_{3} & =0 \\
Q_{1}{ }^{2}+Q_{2}{ }^{2}+Q_{3}{ }^{2}+Q_{4}{ }^{2}-1 & =0
\end{aligned}
$$

\section{Singularity Analysis with Grassmann-Cayley Algebra}

\subsection{Grassmann-Cayley Algebra}

The Grassmann-Cayley Algebra (GCA), also known as exterior algebra, was developed by H. Grassmann as a calculus for linear varieties operating on extensors with the join and meet operators. The latter are associated with the span and intersec- 
tion of vector spaces of extensors. Extensors are symbolically denoted by Plücker coordinates of lines and characterized by their step. In the four-dimensional vector space $V$ associated with the three-dimensional projective space $P_{3}$, extensors of step 1, 2 and 3 represent points, lines and planes, respectively. They are also associated with subspaces of $V$, of dimension 1, 2 and 3, respectively. Points are represented with their homogeneous coordinates, while lines and planes are represented with their Plücker coordinates. The notion of extensor makes it possible to work at the symbolic level and therefore, to produce coordinate-free algebraic expressions for the geometric singularity conditions of spatial parallel manipulators (PMs). For further details on GCA, the reader is referred to $[6,10,11,12,13]$.

\subsection{Twist System of the 3-PPPS Manipulator}

A unit screw is given by $\hat{\$}=\left[\mathbf{s},\left(\mathbf{s}_{\mathbf{0}} \times \mathbf{s}+\lambda \mathbf{s}\right)\right]^{T}$ where $\mathbf{s}$ is a unit vector along the screw axis, $\mathbf{s}_{\mathbf{0}}$ is the position vector of a point on the screw axis, with respect to a reference frame and $\lambda$ is the pitch of the screw. A screw of intensify $\rho$ is written as: $\$=\rho \hat{\$}$. A zero pitch screw $\hat{\$}_{0}=(\mathbf{s}, \mathbf{r} \times \mathbf{s})^{T}(\lambda=0)$ corresponds to the Plücker coordinate vector of a finite line in $P_{3}$. An infinite pitch screw $\hat{\$}_{\infty}=(\mathbf{0}, \mathbf{s})^{T}(\lambda \rightarrow \infty)$ corresponds to the Plücker coordinate vector of an infinite line in the projective space $P_{3}$.

A twist is a screw representing the instantaneous motion of a rigid body, a wrench is a screw representing a system of forces and moments acting on a rigid body. Let $\varepsilon$ denote a twist and $\tau$ denote a wrench.

(a) An infinite pitch twist $\varepsilon_{\infty}$ represents a pure translation;

(b) A zero pitch twist $\varepsilon_{0}$ represents a pure rotation;

(c) A pure force constrains the translation along its line of action and is represented by a zero pitch wrench $\tau_{0}$;

(d) A pure moment constrains the rotation about its direction and is represented by an infinite pitch wrench $\tau_{\infty}$.

Each leg of the 3-PPPS manipulator provides three independent translations and three independent rotations, represented with six independent twists $\hat{\varepsilon}_{\infty 1}^{i}, \hat{\varepsilon}_{\infty 2}^{i}, \hat{\varepsilon}_{\infty 3}^{i}$, $\hat{\varepsilon}_{01}^{i}, \hat{\varepsilon}_{02}^{i}, \hat{\varepsilon}_{03}^{i}, i=1,2,3$, that span its twist sytem T ${ }^{i}$. As shown in Fig. 1, the independent twists can be defined as:

$$
\hat{\varepsilon}_{01}^{i}=\left[\begin{array}{c}
\mathbf{u} \\
\mathbf{c}_{i} \times \mathbf{u}
\end{array}\right], \hat{\varepsilon}_{02}^{i}=\left[\begin{array}{c}
\mathbf{v} \\
\mathbf{c}_{i} \times \mathbf{v}
\end{array}\right], \hat{\varepsilon}_{03}^{i}=\left[\begin{array}{c}
\mathbf{w} \\
\mathbf{c}_{i} \times \mathbf{w}
\end{array}\right]
$$

and

$$
\begin{aligned}
& \hat{\varepsilon}_{\infty 2}^{1}=\hat{\varepsilon}_{\infty 3}^{2}=\hat{\varepsilon}_{\infty 1}^{3}=[\mathbf{0}, \mathbf{u}]^{T} \\
& \hat{\varepsilon}_{\infty 1}^{1}=\hat{\varepsilon}_{\infty 2}^{2}=\hat{\varepsilon}_{\infty 3}^{3}=[\mathbf{0}, \mathbf{v}]^{T} \\
& \hat{\varepsilon}_{\infty 3}^{1}=\hat{\varepsilon}_{\infty 1}^{2}=\hat{\varepsilon}_{\infty 2}^{3}=[\mathbf{0}, \mathbf{w}]^{T}
\end{aligned}
$$


where $\mathbf{c}_{1}, \mathbf{c}_{2}$ and $\mathbf{c}_{3}$ are the Cartesian coordinate vectors of points $C_{1}, C_{2}$ and $C_{3}$, respectively; $\mathbf{u}, \mathbf{v}$ and $\mathbf{w}$ are the unit vectors along the $X, Y$ and $Z$ axes of $\mathscr{F}_{b}$.

$$
\mathrm{T}=\bigcap_{i=1}^{3} \mathrm{~T}^{i}
$$

\subsection{Wrench System of the 3-PPPS Manipulator}

The actuated joints of the 3-PPPS manipulator are the first two prismatic joints of each leg. The actuation wrench $\hat{\tau}_{01}^{i}$ corresponding to the first prismatic joint of the $i$ th leg is reciprocal to all the twists in $\mathrm{T}^{i}$, but to $\hat{\varepsilon}_{\infty 1}^{i}$. Likewise, the actuation wrench $\hat{\tau}_{02}^{i}$ corresponding to the second prismatic joint of the $i$ th leg is reciprocal to all the twists in $\mathrm{T}^{i}$, but to $\hat{\varepsilon}_{\infty 2}^{i}$. As a result,

$$
\hat{\tau}_{01}^{1}=\left[\begin{array}{c}
\mathbf{v} \\
\mathbf{c}_{1} \times \mathbf{v}
\end{array}\right], \hat{\tau}_{01}^{2}=\left[\begin{array}{c}
\mathbf{w} \\
\mathbf{c}_{2} \times \mathbf{w}
\end{array}\right], \hat{\tau}_{01}^{3}=\left[\begin{array}{c}
\mathbf{u} \\
\mathbf{c}_{3} \times \mathbf{u}
\end{array}\right]
$$

and

$$
\hat{\tau}_{02}^{1}=\left[\begin{array}{c}
\mathbf{u} \\
\mathbf{c}_{1} \times \mathbf{u}
\end{array}\right], \hat{\tau}_{02}^{2}=\left[\begin{array}{c}
\mathbf{v} \\
\mathbf{c}_{2} \times \mathbf{v}
\end{array}\right], \hat{\tau}_{02}^{3}=\left[\begin{array}{c}
\mathbf{w} \\
\mathbf{c}_{3} \times \mathbf{w}
\end{array}\right]
$$

In a non-singular configuration, the six actuation wrenches $\hat{\tau}_{01}^{1}, \hat{\tau}_{02}^{1}, \hat{\tau}_{01}^{2}, \hat{\tau}_{02}^{2}, \hat{\tau}_{01}^{3}$ and $\hat{\tau}_{02}^{3}$ span the actuation wrench system of the 3-PPPS manipulator. As the 3PPPS manipulator does not have any constraint wrench, its global wrench system amounts to its actuation wrench, namely,

$$
\mathrm{W}_{3-\underline{P P P S}}=\operatorname{span}\left(\hat{\tau}_{01}^{1}, \hat{\tau}_{02}^{1}, \hat{\tau}_{01}^{2}, \hat{\tau}_{02}^{2}, \hat{\tau}_{01}^{3}, \hat{\tau}_{02}^{3}\right)
$$

The legs of the 3-PPPS manipulator apply six actuation forces to its movingplatform. Its global wrench system is a six-system. A parallel singularity occurs when the wrenches in the six-system become linearly dependent and span a $k$-system with $k<6$.

\subsection{Wrench Diagram of the 3-PPPS Manipulator in $P_{3}$}

The six actuation forces $\hat{\tau}_{01}^{1}, \hat{\tau}_{02}^{1}, \hat{\tau}_{01}^{2}, \hat{\tau}_{02}^{2}, \hat{\tau}_{01}^{3}$ and $\hat{\tau}_{02}^{3}$ form a basis of the global wrench system $\mathrm{W}_{3}-P P P S$. Those wrenches are represented by six finite lines in $P_{3}$. To obtain the six extensors of the 3-PPPS superbracket, we have to select twelve projective points on the six projective lines, i.e., two points on each line. The extensor of a finite line can be represented by either two distinct finite points or one finite point and one infinite point. 
Let $c_{1}, c_{2}, c_{3}$ be the intersection points of $\hat{\tau}_{01}^{1}$ and $\hat{\tau}_{02}^{1}, \hat{\tau}_{01}^{2}$ and $\hat{\tau}_{02}^{2}, \hat{\tau}_{01}^{3}$ and $\hat{\tau}_{02}^{3}$, respectively. From Fig. 1, $\hat{\tau}_{02}^{1}$ and $\hat{\tau}_{01}^{3}$ are parallel and intersect at the infinite plane $\Pi_{\infty}$ at point $\underline{x}=(\mathbf{x}, 0)^{T}$, which corresponds to the $X$ direction. Likewise, $\hat{\tau}_{01}^{1}$ and $\hat{\tau}_{02}^{2}$ are parallel and intersect at the infinite plane $\Pi_{\infty}$ at point $\mathrm{y}=(\mathbf{y}, 0)^{T}$, which corresponds to the $Y$ direction. Similarly, $\hat{\tau}_{01}^{2}$ and $\hat{\tau}_{02}^{3}$ are parallel and intersect at the infinite plane $\Pi_{\infty}$ at point $\underline{z}=(\mathbf{z}, 0)^{T}$, which corresponds to the $Z$ direction.

Therefore, the six points have been selected to express the 3-PPPS superbracket and $\hat{\tau}_{01}^{1} \equiv \mathrm{c}_{1} \underline{\mathrm{y}}, \hat{\tau}_{02}^{1} \equiv \mathrm{c}_{1} \underline{\underline{x}}, \hat{\tau}_{01}^{2} \equiv \mathrm{c}_{2} \underline{\underline{z}}, \hat{\tau}_{02}^{2} \equiv \mathrm{c}_{2} \underline{\mathrm{y}}, \hat{\tau}_{01}^{3} \equiv \mathrm{c}_{3} \underline{\underline{x}}$ and $\hat{\tau}_{02}^{3} \equiv \mathrm{c}_{3} \underline{\underline{z}}$. The wrench diagram of the 3-PPPS manipulator is shown in Fig. 2.

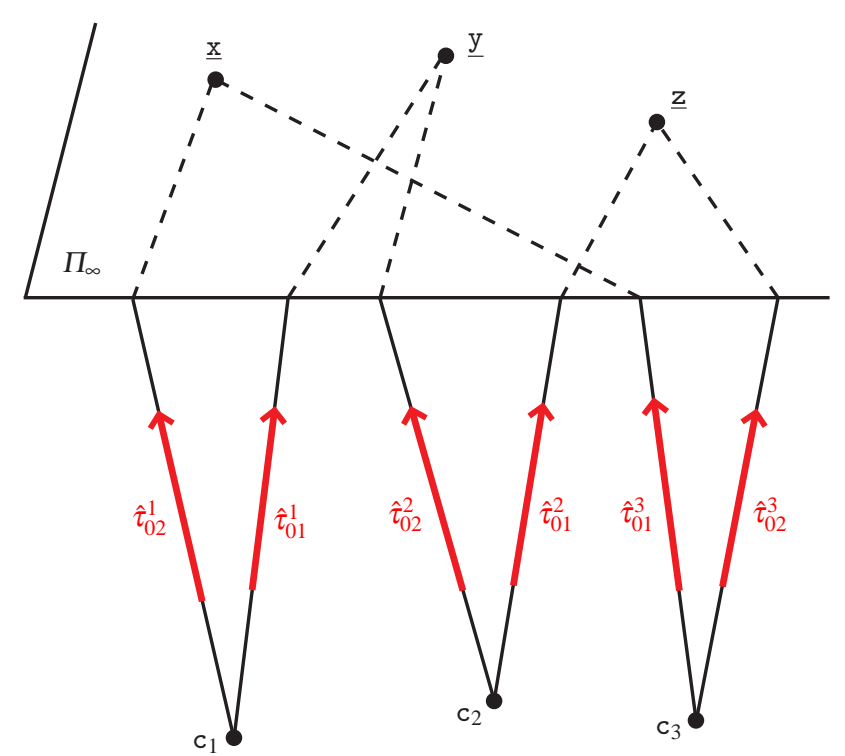

Fig. 2 Wrench diagram in $P_{3}$ of the 3-PPPS manipulator

\subsection{Superbracket of the 3-PPPS Manipulator}

The rows of the backward Jacobian matrix of a parallel manipulator are the Plücker coordinates of six lines in $P_{3}$. The superjoin of these six vectors in $P_{5}$ corresponds to the determinant of their six Plücker coordinate vectors up to a scalar multiple, which is the superbracket in GCA $\Lambda\left(V^{(2)}\right)$ [12]. Thus, a singularity occurs when these six Plücker coordinate vectors are dependent, which is equivalent to a superbracket equal to zero.

The expression of the 3-PPPS superbracket is $\left[c_{1} \underline{\underline{x}} c_{1} \underline{y} c_{2} \underline{y} c_{2} \underline{z} c_{3} \underline{x} c_{3} \underline{z}\right]$, which corresponds to six points selected in the robot wrench diagram. This expression can 
be developed into a linear combination of 24 bracket monomials [6, 14], each one being the product of three brackets of four projective points. The 3-PPPS superbracket was simplified by means of the user interface developed in [15]:

$$
\begin{aligned}
{\left[c_{1} \underline{x} c_{1} \underline{y} c_{2} \underline{y} c_{2} \underline{z} c_{3} \underline{x} c_{3} \underline{z}\right]=} & {\left[c_{3} \underline{x} \underline{z} \underline{y}\right]\left(\left[c_{3} c_{2} \underline{z} c_{1}\right]\left[c_{2} \underline{x} c_{1} \underline{y}\right]-\right.} \\
& {\left.\left[c_{3} \underline{x} \underline{z} c_{2}\right]\left[c_{3} c_{2} \underline{y} c_{1}\right]\right) }
\end{aligned}
$$

\subsection{Geometric Condition for the 3-PPPS Manipulator Singularities}

Let $\Pi_{1}$ be the plane passing through point $C_{1}$ and normal to vector $\mathbf{w}$. Let $\Pi_{2}$ be the plane passing through point $C_{2}$ and normal to vector $\mathbf{u}$. Let $\Pi_{3}$ be the plane passing through point $C_{3}$ and normal to vector $\mathbf{v}$. Let $\Pi_{4}$ be the plane passing through points $C_{1}, C_{2}$ and $C_{3}$. From Eq. (12) and the user interface developed in [15], it turns out that the 3-PPPS manipulator reaches a singular configuration if and only if planes $\Pi_{1}, \Pi_{2}, \Pi_{3}$ and $\Pi_{4}$ intersect at least at one point.

\section{Singularity Analysis with Gröbner Bases}

In this section, we focus on the computation of the parallel singularities of the 3PPPS manipulator using the Jacobian and Gröbner bases. We derive the algebraic relations of the singularities satisfied by the orientation variables.

\subsection{Jacobian Formulation}

The formula we use to define the parallel singularities is the determinant of a Jacobian matrix. This criterion was introduced in [16], where parallel singularities were referred to singularities of the first type. Equations (5a)-(g) depend on six joint variables $\mathbf{T}=\left(x_{1}, y_{1}, y_{2}, z_{2}, x_{3}, z_{3}\right)$, six pose variables $\left(c_{x}, c_{y}, c_{z}, Q_{2}, Q_{3}, Q_{4}\right)$ and one passive variable $\left(Q_{1}\right)$. We denote by $\mathbf{X}$ the union of the pose and the passive variables. Let $\mathbf{A}$ be the Jacobian matrix of these seven equations with respect to $\mathbf{X}$, i.e., 


$$
\begin{aligned}
& \mathbf{A}=\left(\frac{\partial F_{i}}{\partial \mathbf{X}}\right)=
\end{aligned}
$$

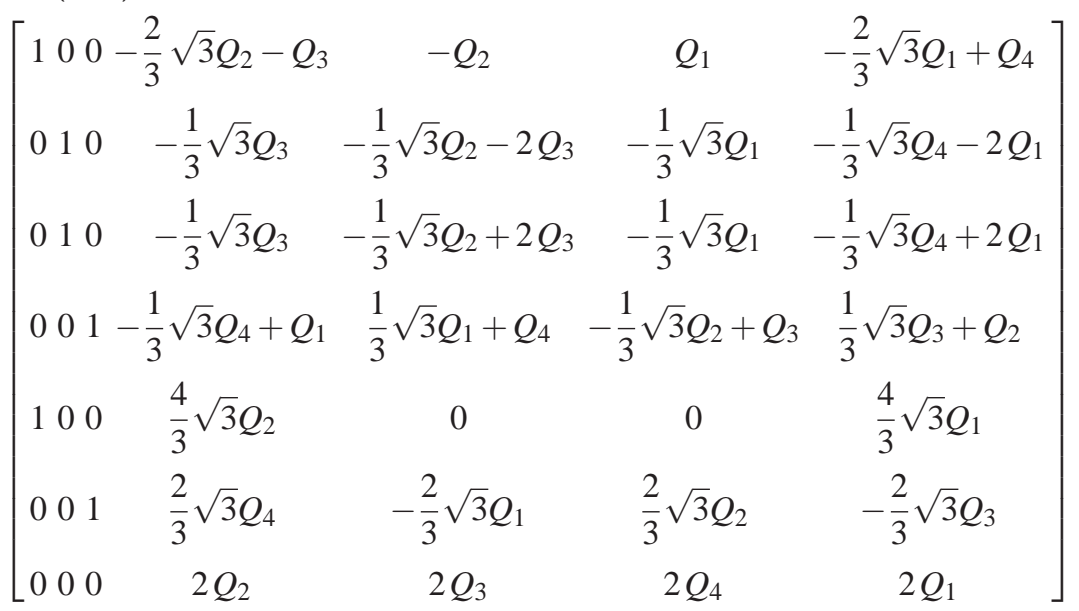

Moreover, let $\mathbf{B}$ be the Jacobian matrix of Eqs. (5a)-(g) with respect to the joint variables. It appears that $\mathbf{B}$ is the negative identity matrix. Denoting by $F(\mathbf{X}, \mathbf{T})$ the vector of seven polynomials on the left-hand side of Eqs. (5a)-(g), we have:

$$
F(\mathbf{X}, \mathbf{T})=0
$$

Differentiating Eq. (14) with respect to time we obtain:

$$
\mathbf{A} \dot{\mathbf{X}}-\dot{\mathbf{T}}=0
$$

In particular, as in [16], we can infer from Eq. (15) that parallel singularities occur when the determinant of $\mathbf{A}$ vanishes:

$$
\begin{aligned}
\operatorname{det}(\mathbf{A})=-8 Q_{4} & \sqrt{3} Q_{3}{ }^{3}+48 Q_{2}{ }^{2} Q_{3} Q_{1}-48 Q_{2} Q_{3}{ }^{2} Q_{4} \\
& -24 \sqrt{3} Q_{2}^{2} Q_{3} Q_{4}+48 Q_{4} Q_{2} Q_{1}^{2}+24 \sqrt{3} Q_{2} Q_{1} Q_{4}{ }^{2} \\
& +24 Q_{3} \sqrt{3} Q_{1}^{2} Q_{4}-48 Q_{3} Q_{1} Q_{4}{ }^{2}-24 Q_{2} Q_{1} \sqrt{3} Q_{3}{ }^{2} \\
& +8 \sqrt{3} Q_{4}{ }^{3} Q_{3}-8 \sqrt{3} Q_{2}{ }^{3} Q_{1}+8 Q_{2} \sqrt{3} Q_{1}{ }^{3}=0
\end{aligned}
$$

Besides, it turns out that the 3-PPPS manipulator does not have any serial singularity as matrix $\mathbf{B}$ is always invertible.

\subsection{Singularities in the Workspace}

The singularities of this mechanism can be represented in terms of the pose variables. To this end, we need to eliminate the joint and passive variables from Eq. (16) and Eqs. (5a)-(g). This can be achieved with methods based on Gröbner basis theory. 


\subsubsection{Gröbner basis for elimination}

Let $\mathscr{P}$ be a set of polynomials in the variables $\left(x_{1}, y_{1}, y_{2}, z_{2}, x_{3}, z_{3}\right)$ and $\left(c_{x}, c_{y}, c_{z}\right.$, $\left.Q_{2}, Q_{3}, Q_{4}\right)$. Moreover, let $\mathscr{V}$ be the set of common roots of the polynomials in $\mathscr{P}$, and let $\mathscr{W}$ be the projection of $\mathscr{V}$ on the workspace. It might not be possible to represent $\mathscr{W}$ by polynomial equations. Let $\overline{\mathscr{W}}$ be the smallest set defined by polynomial equations that contains $\mathscr{W}$. Our goal is to compute the polynomial equations defining $\overline{\mathscr{W}}$.

These polynomial equations are computed with Gröbner-basis theory. A Gröbner basis of a polynomial system is a polynomial system equivalent to the first one, and satisfying some additional specific properties. The Gröbner basis of a system depends on an ordering on the monomials. In our case, if we choose an elimination ordering eliminating $\mathbf{X}$, then the Gröbner basis of $\mathscr{P}$ will contain exactly the polynomials defining $\overline{\mathscr{W}}$. This theory is not the subject of this article and shall not be further detailed here. An introduction on elimination with Gröbner basis is available in [17, Chapter 3].

\subsubsection{Equations of the parallel singularities in the workspace}

We can now use the elimination of the previous paragraph for our problem to obtain the polynomial equations defining implicitly the parallel singularities in the workspace. Let us consider the polynomial set:

$$
\left\{\begin{aligned}
F(\mathbf{X}, \mathbf{T}) & =[0,0,0,0,0,0,0]^{T} \\
\operatorname{det}(\mathbf{A}) & =0
\end{aligned}\right.
$$

We compute a Gröbner basis of system (17) with respect to elimination ordering eliminating $\mathbf{X}$. This computation yields directly the relation satisfied by the parallel singularities in the orientation workspace, namely,

$$
\begin{aligned}
& -Q_{2}{ }^{2}+9 Q_{2}{ }^{2} Q_{4}{ }^{2}+5 Q_{2}{ }^{4}+9 Q_{3}{ }^{2} Q_{2}{ }^{2} \\
& +16 Q_{3}{ }^{6} Q_{2}{ }^{2}+44 Q_{3}{ }^{4} Q_{2}{ }^{4}+32 Q_{3}{ }^{2} Q_{2}{ }^{6}-24 Q_{3}{ }^{4} Q_{2}{ }^{2} \\
& -40 Q_{3}{ }^{2} Q_{2}{ }^{4}+16 Q_{4}{ }^{6} Q_{3}{ }^{2}+28 Q_{4}{ }^{4} Q_{3}{ }^{4}+16 Q_{4}{ }^{2} Q_{3}{ }^{6} \\
& +16 Q_{4}{ }^{6} Q_{2}{ }^{2}+48 Q_{4}{ }^{4} Q_{3}{ }^{2} Q_{2}{ }^{2}+72 Q_{4}{ }^{2} Q_{3}{ }^{4} Q_{2}{ }^{2}+20 Q_{4}{ }^{4} Q_{2}{ }^{4} \\
& +64 Q_{4}{ }^{2} Q_{3}{ }^{2} Q_{2}{ }^{4}+8 Q_{4}{ }^{2} Q_{2}{ }^{6}-24 Q_{4}{ }^{4} Q_{3}{ }^{2}-24 Q_{4}{ }^{2} Q_{3}{ }^{4} \\
& -48 Q_{4}{ }^{2} Q_{3}{ }^{2} Q_{2}{ }^{2}-16 Q_{4}{ }^{2} Q_{2}{ }^{4}+9 Q_{4}{ }^{2} Q_{3}{ }^{2}+16 Q_{4}{ }^{2} \sqrt{3} Q_{3} Q_{2} \\
& +40 \sqrt{3} Q_{4}{ }^{4} Q_{3}{ }^{3} Q_{2}+48 \sqrt{3} Q_{4}{ }^{2} Q_{3}{ }^{5} Q_{2}+40 \sqrt{3} Q_{4}{ }^{4} Q_{3} Q_{2}{ }^{3}+80 \sqrt{3} Q_{4}{ }^{2} Q_{3}{ }^{3} Q_{2}{ }^{3} \\
& +32 \sqrt{3} Q_{4}{ }^{2} Q_{3} Q_{2}{ }^{5}-16 \sqrt{3} Q_{4}{ }^{4} Q_{3} Q_{2}-60 \sqrt{3} Q_{4}{ }^{2} Q_{3}{ }^{3} Q_{2}-52 \sqrt{3} Q_{4}{ }^{2} Q_{3} Q_{2}{ }^{3} \\
& -8 Q_{2}{ }^{6}+4 Q_{2}{ }^{8}-4 \sqrt{3} Q_{2}{ }^{3} Q_{3}-16 \sqrt{3} Q_{3}{ }^{5} Q_{2}{ }^{3} \\
& -24 \sqrt{3} Q_{3}{ }^{3} Q_{2}{ }^{5}-8 \sqrt{3} Q_{3} Q_{2}{ }^{7}+20 \sqrt{3} Q_{3}{ }^{3} Q_{2}{ }^{3}+12 \sqrt{3} Q_{3} Q_{2}{ }^{5} \\
& -24 Q_{4}{ }^{4} Q_{2}{ }^{2}=0
\end{aligned}
$$


We can notice that these equations depend only on the orientation variables $\left(Q_{2}, Q_{3}\right.$, $Q_{4}$ ). This means that the parallel singularities do not depend on the position of the centroid of the moving platform. As a matter of fact, the parallel singularities of the 3-PPPS manipulator can be represented in its orientation workspace only, the latter being characterized with variables $\left(Q_{2}, Q_{3}, Q_{4}\right)$ as shown in Fig. 3 .
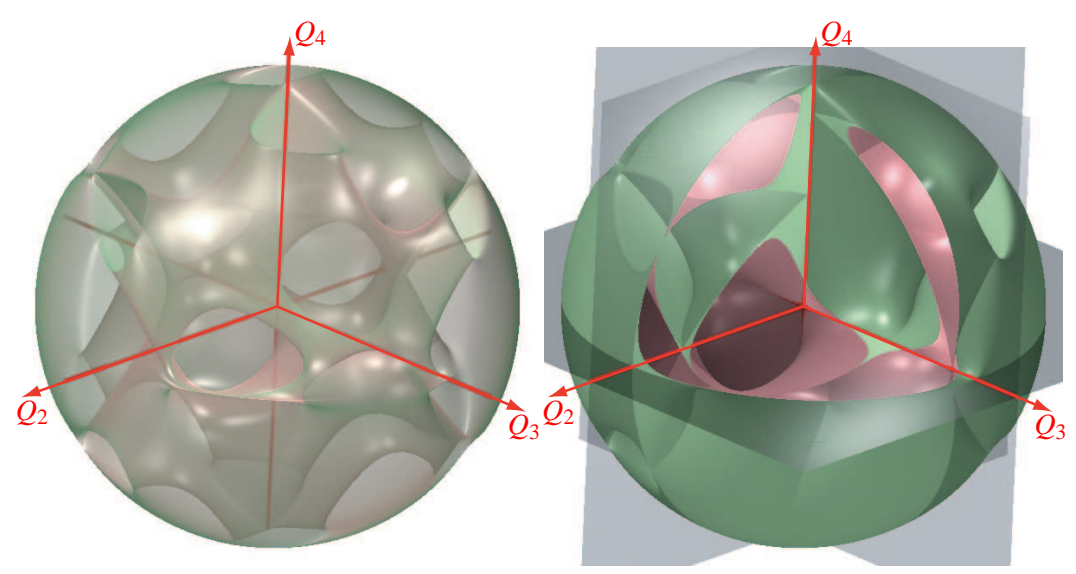

Fig. 3 The parallel singularities of the 3-PPPS manipulator in the orientation workspace: given a point $\mathrm{M}$, vector $\overrightarrow{O M}$ defines the orientation axis and its Euclidean norm $\|\overrightarrow{O M}\|$ is the sine of the half-angle of rotation

Finally, note that Eq. (18) was also obtained from the geometric condition for the 3-PPPS manipulator singularities given in Sec. 3.6.

\section{Conclusions}

The subject of this paper was the singularity analysis of a six-dof three-legged parallel mechanism to be used as a force-feedback interface. A geometric condition for the manipulator singularities was obtained by means of Grassmann-Cayley algebra. The parallel singularities of the manipulator were computed using the Jacobian and Gröbner bases. Consequently, the algebraic relations of the singularities satisfied by the orientation variables were derived. Finally, the parallel singularities of the manipulator were plotted in its orientation workspace. 


\section{Acknowledgments}

This work has been supported by the "Agence Nationale de la Recherche (ANR SIROPA)".

\section{References}

1. M.G. Alonso, F. Vexo and D. Thalmann, Stepping into Virtual Reality, London: Springer, 2008.

2. G. Burdea, Force and Touch Feedback for Virtual Reality, New York: John Wiley and Sons, 1996

3. B. Siciliano and O. Khatib, Handbook of Robotics, Springer, 2008.

4. C. Chen, D. Jackson and W. Heyne. "A new 6-dof 3-legged parallel mechanism for forcefeedback interface". Proceeding of the 2010 IEEE/ASME International Conference on Mechatronic and Embedded Systems and Applications, July, Qingdao, 2010.

5. J.P. Merlet. Singular Configurations of Parallel Manipulators and Grassmann Geometry. The International Journal of Robotics Research, 8: pp. 45-56, June 1989.

6. P. Ben-Horin and P. Shoham. Singularity Condition of Six-Degree-Of-Freedom ThreeLegged Parallel Robots Based on Grassmann-Cayley Algebra. IEEE transactions on robotics, 22: pp. 577-590, 2006

7. P. Ben-Horin and P. Shoham. Application of Grassmann-Cayley Algebra to Geometrical Interpretation of Parallel Robot Singularities. International Journal of Robotics Research, 1: pp. 127-141, 2009.

8. D. Kanaan, P. Wenger, S. Caro, and D. Chablat. Singularity Analysis of Lower-Mobility Parallel Manipulators Using Grassmann-Cayley Algebra. IEEE Transactions on Robotics, 25: pp. 995-1004, 2009.

9. W. Khalil and E. Dombre, Modeling, identification and control of robots, Kogan Page Science, 2004.

10. N. L. White. The Bracket Ring of a Combinatorial Geometry I. Transactions of the American Mathematical Society, 202, pp. 79-95, 1975.

11. N. L. White. The bracket of 2-extensors. Congressus Numerantium, 40, pp. 419-428, 1983.

12. N. L. White. Grassmann-Cayley Algebra and Robotics Applications. Handbook of Geometric Computing, Part VIII, pp. 629-656, 2005.

13. S. Amine, D. Kanaan, S. Caro and P. Wenger. "Singularity Analysis of Lower-Mobility Parallel Robots with an Articulated Nacelle". On Advances in Robot Kinematics, Springer, pp. $273-282,2010$

14. T. McMillan and N.L. White The Dotted Straightening Algorithm Journal of Symbolic Computation, 11: pp. 471-482, 1991.

15. P. Ben-Horin, M. Shoham, S. Caro, D. Chablat and P. Wenger. "User Interface for the Singularity Analysis of Parallel Robots Based on GrassmannCayley Algebra". On Advances in Robot Kinematics, Springer, pp. 49-58, 2008.

16. C. Gosselin and J. Angeles, "Singularity analysis of closed-loop kinematic chains", IEEE Journal of Robotics and Automation, Vol. 6, No. 3, pp. 281-290, 1990.

17. D. Cox, J. Little and D. O'Shea, Ideals, Varieties, and Algorithms, Undergraduate Texts in Mathematics. Springer Verlag, 1992. 